\title{
The Association Between Serum Thyrotropin Within the Reference Range and Metabolic Syndrome in a Community-Based Chinese Population
}

This article was published in the following Dove Press journal: Diabetes, Metabolic Syndrome and Obesity: Targets and Therapy

\author{
Meng Li $\mathbb{D}^{1, *}$ \\ Xiuying Zhang ${ }^{1} *$ \\ Xianghai Zhou' \\ Xueyao Han' \\ Rui Zhang' \\ Zuodi Fu \\ Lianying Wang ${ }^{2}$ \\ Ying Gao' \\ Yufeng $\mathrm{Li}^{2}$ \\ Linong $\mathrm{Ji}^{1}$ \\ 'Department of Endocrinology and \\ Metabolism, Peking University People's \\ Hospital, Peking University Diabetes \\ Center, Beijing, People's Republic of \\ China; ${ }^{2}$ Department of Endocrinology \\ and Metabolism, Capital Medical \\ University Pinggu Hospital, Beijing, \\ People's Republic of China
}

*These authors contributed equally to this work
Purpose: We aimed to ascertain the association between thyrotropin (TSH) levels in euthyroid state and the prevalence of metabolic syndrome (MetS) in a community-based Chinese population.

Participants and Methods: Based on a large and well-characterized community cohort in Beijing, China, 1831 men and 1742 women with serum TSH levels within the reference range $(0.50-4.78 \mu \mathrm{IU} / \mathrm{mL})$ were stratified by quartiles of TSH (Q1-4). MetS was identified according to the criteria of International Diabetes Federation guidelines. Poisson regression models were used to estimate the association between serum TSH and the prevalence of MetS and its components before and after adjustment for potential confounding factors. The reported association was measured using the prevalence ratio (PR) with its respective 95\% confidence interval $(95 \% \mathrm{CI})$.

Results: The prevalence of MetS in euthyroid population across TSH quartiles (Q1-4) was $38.9 \%, 44.6 \%, 41.0 \%$, and $47.7 \%$, respectively, in men $(P=0.045)$, and $47.7 \%, 46.6 \%$, $46.9 \%$, and $54.6 \%$, respectively, in women $(P=0.032)$. Compared with the reference group TSH-Q1, the prevalence of MetS was higher among TSH-Q4 group both in men ( $\mathrm{PR}=1.27$; 95\% CI: $1.09,1.48, P=0.002)$ and women ( $\mathrm{PR}=1.21 ; 95 \% \mathrm{CI}: 1.07,1.37, P=0.003)$ even after adjustment for age, lifestyle factors, serum levels of free triiodothyronine (FT3), and free thyroxine (FT4). Most of the components of MetS were common in higher serum TSH levels within the normal range.

Conclusion: The prevalence of MetS and most of its components increased in the higher TSH group in euthyroid Chinese population.

Keywords: thyroid hormones, obesity, hypertension, hyperglycemia

\section{The Plain Language Summary}

- Why was the Study Done?

Department of Endocrinology and Metabolism, Peking University People's

Hospital, Beijing 100044, People's

Republic of China

Tel/Fax +86- |0-8832437|

Email jiln@bjmu.edu.cn

Yufeng $\mathrm{Li}$

Department of Endocrinology and

Metabolism, Capital Medical University

Pinggu Hospital, Beijing 101200, People's

Republic of China

$\mathrm{Tel} / \mathrm{Fax}+86-10-89978790$

Email doctorlyf@I26.com
Metabolic syndrome (MetS) is increasing throughout the world, and thyroid function has an influence on systematic metabolism. However, the association between MetS and thyrotropin (TSH) was elusive. We aimed to investigate the association between MetS and TSH in euthyroid subjects from a large community-based Chinese population.

- What Did the Researchers Do?

We performed a large and well-characterized community cohort in Pinggu (PG), Beijing, China. The PG cohort is to study the course and pathogenesis of metabolic diseases. During 
the survey, we have collected detailed demographic and clinical, biochemical data, and determined the strata of glucose homeostasis, lipid metabolism and thyroid function.

- What Did We Find?

Based on the database of community-based population, we found that the prevalence of MetS and most of its components increased in the higher TSH group in euthyroid Chinese population.

- What Do These Results Mean?

The assessment of thyroid function may contribute to early identification of chronic metabolic diseases and initiate personalized treatment strategies. Further research into the causality on the association between TSH and MetS is to be warranted.

\section{Introduction}

Metabolic syndrome (MetS) representing a cluster of metabolic abnormalities that include abdominal obesity, hyperglycemia, hypertension, hypertriglyceride and lowhigh-density lipoprotein cholesterol (HDL-C) levels has increased all over the world and become a major public health care concern. ${ }^{1,2}$ Thyroid hormone, as one of the most fundamental hormones in humans, is the root of systemic metabolism, energy balance and cardiovascular function., ${ }^{3,4}$ Mounting studies have shown that overt hypothyroidism and subclinical hypothyroidism have association with an increased risk for hypertension, obesity, dyslipidemia, diabetes, cardiovascular disease and mortality. ${ }^{4-9}$ However, whether the level of thyroid function in the normal range is related to MetS and to what extent remain inconclusive. The HUNT Study in Norway and the DanThyr Study in Denmark have reported the association between higher thyrotropin (TSH) levels within the reference range and the presence of obesity, less favorable lipid profiles and high blood pressure. ${ }^{10-12}$ Some studies also reported that subjects with high normal TSH had an increased likeliness of MetS. ${ }^{13-15}$ However, Mehran et al failed to find the association between TSH and MetS. ${ }^{16}$ The studies on the association between them in Chinese population are mostly conducted among healthy examination populations, with inconsistent results. Recently, in a large population taking healthy examinations in Taiwan, elevated TSH levels within the normal range were identified as a cardiometabolic risk marker associated with MetS and other metabolic risk factors. ${ }^{14}$ In contrast, Huang et al found no significant correlation between TSH and MetS after adjustment of confounders (smoking and age) in euthyroid Taiwanese adults. ${ }^{17}$ Another study conducted among Chinese adolescents did not find any correlation between the prevalence of MetS and its components and normal TSH. ${ }^{18}$ Two other Chinese studies identified the relationship between TSH and MetS components, ${ }^{19,20}$ and they did not consider MetS as a whole in the study conducted in a southwest city. ${ }^{19}$ As we know, the definitions of MetS were distinct in these studies. In addition, the research design, different subjects, reference range of $\mathrm{TSH}$, and the potential confounders were also attributed to the conflicting results. To date, there are few studies addressing the association between $\mathrm{TSH}$ in the normal range and the prevalence of MetS in Chinese general community-based population.

As community-based population, the Pinggu (PG) cohort was established in northern China to study the course and pathogenesis of metabolic diseases. During the cohort survey, we have collected detailed epidemic, clinical and biochemical data, and determined the strata of glucose homeostasis, lipid metabolism and thyroid function. Here, we tried to investigate whether the serum TSH levels within the reference range were associated with the prevalence of MetS and its components, including central obesity, high blood pressure, hypertriglyceride, low-HDL$\mathrm{C}$ and hyperglycemia.

\section{Participants and Methods Study Population}

Participants of this study were from a community-based cohort established for the Pinggu Metabolic Disease Study (PMDS) in Pinggu district of Beijing, northern China from September 2013 to July 2014. The protocol has been described previously. ${ }^{21,22}$ Briefly, based on the national Civil Registration system, a total of 6583 PG participants aged 26-76 years were randomly selected according to gender and age compositions, and finally 4002 individuals finished the survey, with a response rate of $60.8 \%$.

In this study, we excluded (1) participants who have received thyroid surgery or thyroid medications (i.e., thyroid hormone, anti-thyroid drugs, or radioiodine therapy) $(\mathrm{N}=115)$; (2) those diagnosed as hyperthyroidism or TSH $<0.55 \mu \mathrm{IU} / \mathrm{mL}$ at the baseline $(\mathrm{N}=74)$; (3) those diagnosed as hypothyroidism or TSH $>4.78 \mu \mathrm{IU} / \mathrm{mL}$ at the baseline ( $\mathrm{N}=237)$; and (4) those taking medications or in other abnormal status that could affect thyroid function $(\mathrm{N}=3)$. After these exclusions, a total of 1831 men and 
1742 women with complete information were analyzed (Figure S1). PMDS was approved by the ethics committee of Peking University Medical Center. All participants gave written informed consent.

\section{Questionnaire and Physical Examination}

All participants accepted face-to-face interview and standardized questionnaires, including demographics, lifestyle, information on history of thyroid disease, diabetes, hypertension, dyslipidemia and related chronic diseases, the associated treatments, and other health-related issues. The smoking status was divided into never smoked, exsmoker or current smoker. In dietary questions, the participants reported the frequency of food intake. Here we choose the frequency of red meat as the parameter of dietary habit and classified as 0 when consumed less than seven times per week, or 1 when consumed more than seven times per week. Alcohol consumption was divided into $0,>0$ and $\leq 140,>140, \leq 210$, and $>210 \mathrm{~g} /$ week in men and $0,>0$ and $\leq 70,>70, \leq 140$, and $>140 \mathrm{~g} /$ week in women. Sedentary time per day was also recorded, and daily sedentary time was categorized into $<1.5,1.5-2.5,2.5-3.5,3.5-4.5$, or $\geq 4.5$ hours $/$ day. ${ }^{23}$ Women were determined to be postmenopausal if their menstrual periods had stopped for at least one year. The studied postmenopausal individuals here did not receive estrogen treatment.

In addition, anthropometric data were collected by trained physicians according to standardized protocols. Height and weight were measured when subjects stood without shoes and light clothing. Body mass index (BMI) was calculated: $\mathrm{BMI}=$ weight $(\mathrm{kg}) /$ height $^{2}\left(\mathrm{~m}^{2}\right)$. Waist circumference (WC) was measured around the abdomen at the middle point between the anterosuperior iliac crest and the inferior margin of 12th ribs. Systolic blood pressure (SBP) and diastolic blood pressure (DBP) were measured three times after a 5-minute rest with the participant in the sitting position, and the mean value of the three measurements was used.

\section{Laboratory Measurements}

Fasting blood samples after overnight were collected. Fasting plasma glucose (FPG) was measured by the glucose oxidase method. Serum total cholesterol, TG, HDLC, low-density lipoprotein cholesterol (LDL-C) and uric acid were measured by an automated clinical chemistry method (UnicelDxC 800; Beckman Coulter, Miami, FL, USA). Haemoglobin A1c (HbAlc) was measured using cation-exchange high-pressure liquid chromatography (Adams A1c HA-8160; Arkray, Kyoto, Japan). Serum insulin was tested by a radioimmunoassay method (China Institute of Atomic Energy, Beijing, China). The thyroid hormones and thyroid-related antibodies were tested using a supersensitive electrochemiluminescence immunoassay (Siemens Centaur XP, Germany). The intra-assay coefficient of variation $(\mathrm{CV})$ was less than $8 \%$, and the interassay CV less than $10 \%$ for all these parameters. The HOMA-IR score was calculated using the following formula: HOMA-IR $=($ FPG $[\mathrm{mmol} / \mathrm{L}] \times \operatorname{insulin}[\mu \mathrm{U} /$ $\mathrm{mL}]) / 22.5$.

\section{Diagnostic and Grouping Criteria}

The reference range for serum thyrotropin (TSH) is $0.55-$ $4.78 \mu \mathrm{IU} / \mathrm{mL}$. The reference intervals were $11.45-23.17$ $\mathrm{pmol} / \mathrm{L}$ for free thyroxine (FT4), $3.50-6.50 \mathrm{pmol} / \mathrm{L}$ for free triiodothyronine (FT3), $<60 \mathrm{IU} / \mathrm{mL}$ for thyroid peroxidase antibodies (TPOAb) and $<15 \mathrm{IU} / \mathrm{mL}$ for thyroglobulin antibody (TGAb). Euthyroidism was defined as serum TSH in the range of $0.55-4.78 \mu \mathrm{IU} / \mathrm{mL}$, regardless of the FT4 concentration. The Pinggu population is considered to have sufficient iodine intake. Participants who considered euthyroid were further divided into four groups: Q1 (TSH $0.55-1.30 \mu \mathrm{IU} / \mathrm{mL}$ ), Q2 (TSH $1.31-1.85 \mu \mathrm{IU} / \mathrm{mL})$, Q3 (TSH $1.85-2.58 \mu \mathrm{IU} / \mathrm{mL}$ ), and Q4 $(2.59-4.78 \mu \mathrm{IU} / \mathrm{mL})$ groups.

Metabolic syndrome was defined according to the International Diabetes Federation (IDF 2005) criteria: $^{24}$ the diagnosis of central obesity (WC $\geq 90 \mathrm{~cm}$ in men or $\geq 80 \mathrm{~cm}$ in women), plus any two of the following four factors: (1) TG levels $\geq 1.7 \mathrm{mmol} / \mathrm{L}$, (2) HDL-C $<1.03$ $\mathrm{mmol} / \mathrm{L}$ in men or $<1.29 \mathrm{mmol} / \mathrm{L}$ in women, (3) $\mathrm{SBP} \geq$ $130 \mathrm{mmHg}$ or DBP $\geq 85 \mathrm{mmHg}$ or previous diagnosis with hypertension, (4) FBG $\geq 5.6 \mathrm{mmol} / \mathrm{L}$, or previous diagnosis with type 2 diabetes mellitus. The components of MetS included central obesity, hypertriglyceride, low-HDL-C levels, hypertension, and hyperglycemia. Obesity was also defined as BMI $\geq 28 \mathrm{~kg} / \mathrm{m}^{2}$ according to the Chinesespecific BMI cut-off point. ${ }^{25}$

\section{Statistical Analysis}

Data processing and statistical analysis were performed with SPSS 21.0 software. Continuous variables were presented as mean with standard deviation (mean $\pm \mathrm{SD}$ ) or median (25th, 75th). Categorical data were presented as number and proportion (\%). Normally distributed variables were compared using $t$-tests, and variables with 
skewed distribution were compared with Mann-Whitney $U$-tests between men and women groups. One-way ANOVA and Kruskal-Wallis tests were used to compare the characteristics across TSH quartiles. A Chi-square was used for categorical data. Generalized linear models from Poisson family were constructed to evaluate the association between TSH quartiles with the prevalence of MetS before and after adjustment for the confounders screening from epidemiological investigation and correlation analysis. The adjusted model 2 included the following confounding variables: age, smoking, drinking, red meat intake, sedentary time, menopause (in women); and the adjusted model 3 further included the variables: FT3 and FT4. The prevalence ratio (PR) and its $95 \%$ confidence interval (CI) were thus reported for the higher TSH levels (Q2-Q4) with the TSH-Q1 as the reference group. The pvalue of $<0.05$ was considered as statistically significant.

\section{Results}

\section{General Population Characteristics}

A total of 3573 participants (1831 men and 1742 women) were finally included in the analysis. The demographic and clinical characteristics are listed in Table 1. Totally, the average age of the analysis population was $49.81 \pm 11.74$ years. The mean BMI was $26.09 \pm 3.83 \mathrm{~kg} / \mathrm{m}^{2}$, with $28.5 \%$ of participants being obesity (BMI $\geq 28 \mathrm{~kg} / \mathrm{m}^{2}$ ). In this study population, $27.7 \%$ of participants were sedentary for $\geq 4.5$ hours per day. The frequency of red meat intake was higher in men than in women. In men, $64.3 \%$ were current smokers and $70.8 \%$ drank. The vast majority of women did not have the habit of smoking and drinking.

The prevalence of MetS was $42.7 \%, 49.4 \%$ and $46.0 \%$ respectively in men, women and the overall study population. As shown in Figure 1A, the prevalence of MetS in men aged 25 to 29,30 to 39,40 to 49,50 to 59 , and $\geq 60$ years was $34.3 \%, 49.2 \%, 47.1 \%, 41.7 \%$, and $35.3 \%(P<$ $0.001)$, respectively, with a very significant trend of early onset. In women, it was $14.8 \%, 19.5 \%, 40.2 \%, 65.7 \%$, and $71.0 \%(P<0.001)$ (Figure 1B), respectively, increasing rapidly after menopause and even exceeding that of men.

\section{The Prevalence of MetS Among Different TSH Quartiles}

The prevalence of Mets in men from TSH-Q1 to TSH-Q4 was $38.9 \%, 44.6 \%, 41.0 \%$, and $47.7 \%(P=0.045)$, respectively (in Table S1a), and in women, it was $47.7 \%, 46.6 \%$, $46.9 \%$, and $54.6 \% \quad(P=0.032)$, respectively (in
Table S1b). As shown in Figure 2, the prevalence of MetS (Figure 2A), obesity and hypertriglyceride (Figure 2C) increased along with the increased TSH levels both in men and women. The prevalence of central obesity and low-HDL-C increased significantly with the elevated TSH levels in men, but not in women (Figure 2B and D). There was no significant difference on the prevalence of hyperglycemia and hypertension among different TSH quartiles (Figure 2E and F). The proportions of positive $\mathrm{TPOAb}$ and TGAb were significantly highest in the TSHQ4 group than other groups (TSH Q1-3) in men $(P=0.018)$ and in women $(P<0.001)$. We examined continuous traits across four TSH groups with $P$-fortrend summarized in Tables S1a and S1b. TSH levels within the normal range were positively associated with age, BMI, waist circumference, TG, and MetS in both men and women. Increased TSH levels showed significant correlations with $\mathrm{HbAlc}$, fasting insulin, HOMA-IR, TC, LDL-C, uric acid (in men), and DBP (in women).

\section{Association Between TSH Quartiles and MetS}

In this analysis, a generalized linear model from Poisson family was adopted to assess the impact of TSH levels on the prevalence of Mets before and after adjustment for potential confounding factors (Table 2). Compared with euthyroid adults with the lowest serum TSH quartile (TSH-Q1), the PRs (95\% CI) of MetS for TSH-Q2, TSHQ3 and TSH-Q4 in men were 1.15 (1.00, 1.32), 1.06 (0.91, $1.24)$ and $1.25(1.08,1.46)$, respectively, and $0.98(0.84$, $1.14), 0.98(0.85,1.14)$ and $1.15(1.00,1.31)$ in women. After adjustments for age, postmenopausal status (for women), smoking status, drinking, red meat intake and sedentary time (model 2), compared with the TSH-Q1, the prevalence of MetS for TSH-Q4 was 23\% (PR 1.23; 95\% CI $1.06,1.43, P=0.007)$ in men and $17 \%$ higher in women (PR $1.17 ; 95 \%$ CI $1.04,1.33, P=0.012$ ). In the fully adjusted model 3 that was further fitted with the levels of serum FT3 and FT4, the trend did not substantially change with higher prevalence of MetS for TSH-Q4 vs TSH-Q1 in men (PR 1.27; 95\% CI 1.09, 1.48, $P=0.002$ ) and women (PR 1.21; 95\% CI 1.07, 1.37, $P=0.003$ ).

After full adjustment for confounders, the prevalence of central obesity in TSH-Q4 was $25 \%$ and $14 \%$ higher compared with TSH-Q1 both in men (PR 1.25; 95\% CI $1.10,1.43, P=0.001$ ) and in women (PR 1.14; $95 \% \mathrm{CI}$ $1.03,1.26, P=0.009)$. The similar trend was shown in the 
Table I Characteristics of the Study Population

\begin{tabular}{|c|c|c|c|c|}
\hline Characteristic & Total & Men & Women & $P$-value \\
\hline Number & 3573 & $|83|$ & 1742 & I \\
\hline Age $(y)$ & $49.81 \pm 11.74$ & $49.66 \pm 11.89$ & $49.97 \pm 11.58$ & 0.430 \\
\hline BMI $\left(\mathrm{kg} / \mathrm{m}^{2}\right)$ & $26.09 \pm 3.83$ & $26.15 \pm 3.75$ & $26.02 \pm 3.90$ & 0.316 \\
\hline Obesity (BMI $\left.\geq 28 \mathrm{~kg} / \mathrm{m}^{2}\right)(\mathrm{n}, \%)$ & $1020(28.5)$ & $532(29.1)$ & $488(28.0)$ & 0.491 \\
\hline WC $(\mathrm{cm})$ & $86.79 \pm 10.86$ & $89.40 \pm 10.24$ & $84.06 \pm 10.81$ & $<0.001$ \\
\hline $\mathrm{SBP}(\mathrm{mmHg})$ & $|3| \pm 18$ & $132 \pm 17$ & $128 \pm 19$ & $<0.001$ \\
\hline $\mathrm{DBP}(\mathrm{mmHg})$ & $79 \pm 11$ & $81 \pm 12$ & $77 \pm 11$ & $<0.001$ \\
\hline $\mathrm{HbAlc}(\%)$ & $5.82 \pm 0.93$ & $5.83 \pm 0.99$ & $5.81 \pm 0.86$ & 0.450 \\
\hline FPG (mmol/L) & $6.07 \pm 1.62$ & $6.25 \pm 1.75$ & $5.88 \pm 1.45$ & $<0.001$ \\
\hline FINS $(\mu \mathrm{IU} / \mathrm{mL})$ & $9.62 \pm 6.32$ & $9.56 \pm 6.74$ & $9.69 \pm 5.85$ & 0.534 \\
\hline HOMA-IR (mU/L mM) & $2.12(1.38,3.26)$ & $2.12(1.29,3.36)$ & $2.12(1.44,3.14)$ & 0.667 \\
\hline TG $(\mathrm{mmol} / \mathrm{L})$ & $1.20(0.77,1.88)$ & $1.29(0.83,2.13)$ & $1.12(0.72,1.66)$ & $<0.001$ \\
\hline TC $(\mathrm{mmol} / \mathrm{L})$ & $4.91 \pm 0.97$ & $4.89 \pm 0.95$ & $4.93 \pm 1.00$ & 0.140 \\
\hline HDL-C (mmol/L) & $1.16 \pm 0.31$ & $1.11 \pm 0.32$ & $1.21 \pm 0.29$ & $<0.001$ \\
\hline LDL-C (mmol/L) & $2.86 \pm 0.80$ & $2.83 \pm 0.79$ & $2.89 \pm 0.81$ & 0.024 \\
\hline $\mathrm{UA}(\mu \mathrm{mol} / \mathrm{L})$ & $286.58 \pm 80.72$ & $324.29 \pm 78.41$ & $246.93 \pm 61.96$ & $<0.001$ \\
\hline FT4 (pmol/L) & $16.05(14.66,17.62)$ & $16.63(15.18,18.24)$ & $15.53(14.23,16.84)$ & $<0.001$ \\
\hline FT3 (pmol/L) & $4.99(4.65,5.35)$ & $5.20(4.88,5.54)$ & $4.78(4.49,5.06)$ & $<0.001$ \\
\hline $\mathrm{TSH}(\mu \mathrm{IU} / \mathrm{mL})$ & $1.85(1.31,2.59)$ & $1.65(1.22,2.33)$ & $2.05(1.48,2.85)$ & $<0.001$ \\
\hline TSH Quartiles (n, \%) & & & & $<0.001$ \\
\hline QI (0.55-1.30) & $885(24.8)$ & $558(30.5)$ & $327(18.8)$ & \\
\hline Q2 (1.3I-1.85) & $906(25.4)$ & $522(28.5)$ & $384(22.0)$ & \\
\hline Q3 (1.85-2.58) & $885(24.8)$ & $407(22.2)$ & $478(27.4)$ & \\
\hline Q4 (2.59-4.78) & $897(25.1)$ & $344(38.4)$ & $553(31.7)$ & \\
\hline Thyroid autoimmune antibodies (n, \%) & & & & $<0.001$ \\
\hline TPOAb and TGAb (-) & $3035(84.9)$ & $1655(90.4)$ & $1380(79.2)$ & \\
\hline TPOAb or TGAb (+) & $337(9.4)$ & $13 \mid(7.2)$ & $206(11.8)$ & \\
\hline TPOAb and TGAb $(+)$ & $201(5.6)$ & $45(2.5)$ & $156(9.0)$ & \\
\hline MetS (n, \%) & $1642(46.0)$ & $781(42.7)$ & $861(49.4)$ & $<0.001$ \\
\hline Central obesity $(\mathrm{n}, \%)$ & $2006(56.1)$ & $908(49.6)$ & $1098(63.0)$ & $<0.001$ \\
\hline Hypertriglyceride (n, \%) & $1051(29.4)$ & $632(34.5)$ & $419(24.1)$ & $<0.001$ \\
\hline Low-HDL-c (n, \%) & $2224(62.2)$ & $1006(54.9)$ & $1218(69.9)$ & $<0.001$ \\
\hline Hypertension (n, \%) & $2187(61.2)$ & $1208(66.0)$ & $979(56.2)$ & $<0.001$ \\
\hline Hyperglycemia (n, \%) & $1908(53.4)$ & $1130(61.7)$ & $778(44.7)$ & $<0.001$ \\
\hline Postmenopausal status (n, \%) & - & - & $850(48.8)$ & l \\
\hline Smoking (n, \%) & & & & $<0.001$ \\
\hline Nonsmoker & $2097(58.7)$ & $373(20.4)$ & $1724(99.0)$ & \\
\hline Ex-smoker & $282(7.9)$ & $280(15.3)$ & $2(0.1)$ & \\
\hline Current smoker & $1194(33.4)$ & $1178(64.3)$ & $16(0.9)$ & \\
\hline Alcohol drinking (g/week) (n, \%) & & & & $<0.001$ \\
\hline 0 & $2100(58.8)$ & $535(29.2)$ & $1565(89.8)$ & \\
\hline $0.1-140$ for men or $0.1-70$ for women & $860(24.1)$ & $728(39.8)$ & $132(7.6)$ & \\
\hline $\mid 40.1-210$ for men or $70.1-140$ for women & $74(2.1)$ & $54(2.9)$ & $20(1.1)$ & \\
\hline$>210$ for men or $>140$ for women & $539(15.1)$ & $514(28.1)$ & $25(1.4)$ & \\
\hline Red meat consumption $\geq 7$ times per week ( $n, \%$ ) & $1424(39.9)$ & $842(46.0)$ & $582(33.4)$ & $<0.001$ \\
\hline Sedentary time (hours/day) (n, \%) & & & & $<0.001$ \\
\hline$<1.5$ & $245(6.9)$ & $120(6.6)$ & $125(7.2)$ & \\
\hline $1.5-2.5$ & $831(23.3)$ & $405(22.1)$ & $426(24.5)$ & \\
\hline
\end{tabular}

(Continued) 
Table I (Continued).

\begin{tabular}{|l|l|l|l|l|}
\hline Characteristic & Total & Men & Women & $P$-value \\
\hline $2.5-3.5$ & $876(24.5)$ & $391(21.4)$ & $485(27.8)$ \\
$3.5-4.5$ & $633(17.7)$ & $312(17.0)$ & $321(18.4)$ & \\
$\geq 4.5$ & $988(27.7)$ & $603(32.9)$ & $385(22.1)$ & \\
\hline
\end{tabular}

Notes: Data are expressed as mean \pm SD for continuous data with normal distribution, median (25th, 75 th percentage) for continuous data with skewed distribution, and $\mathrm{n}$ (\%) for categorical data. $P$-value from $t$-test, chi-squared or Mann-Whitney $U$-test comparing between men and women. $P$-value $<0.05$ was considered significant and in boldface.

Abbreviations: BMI, body mass index; WC, waist circumference; SBP, systolic blood pressure; DBP, diastolic blood pressure; HbAIc, haemoglobin AI c; FPG, fasting plasma glucose; FINS, fasting insulin; HOMA-IR, homeostasis model assessment of insulin resistance; TG, triglycerides; TC, total cholesterol; HDL-C, high-density lipoprotein cholesterol; LDL-C, low-density lipoprotein cholesterol; UA, uric acid; FT4, free thyroxine; FT3, free triiodothyronine; TSH, thyrotropin; Q, quartile; TPOAb, thyroid peroxidase antibody; TGAb, thyroglobulin antibody; MetS, metabolic syndrome.

prevalence of hypertriglyceride (men PR 1.38; 95\% CI $1.15,1.66, P=0.001$, and women PR 1.43; 95\% CI $1.12,1.82, P=0.004)$. The prevalence of hypertension in TSH-Q4 was higher than TSH-Q1 only in women (PR $1.20 ; 95 \%$ CI $1.08,1.33, P=0.001)$. Compared with the TSH-Q1 group, the association between TSH quartiles and hyperglycemia and low-HDL-c in TSH-Q4 were not statistically significant both in men and women.

\section{Discussion}

The results of the current cross-sectional population study in euthyroid adults demonstrated a significant association between serum TSH and the prevalence of MetS. Overall, even if thyroid function is in the normal range, the prevalence of MetS and its components increased from low to high TSH quartiles.

Although numerous studies have explored the association between TSH and the prevalence of MetS, the conclusions are inconsistent. In a large population-based study involving 24,765 euthyroid subjects who underwent health examinations from Taiwan, TSH levels showed a positive association with the presence of MetS. ${ }^{14}$ The prevalence of MetS was significantly higher in subjects with TSH levels $>2.5 \mathrm{mU} / \mathrm{L}$ than the low-TSH group in German and Korean studies. ${ }^{15,26}$ In addition, Zhou et al performed a 7.2-year longitudinal study and found the TSH levels were associated with the development of MetS. ${ }^{27}$ These findings are supported by the results of the current study. Compared with euthyroid adults with the lowest serum TSH quartile, the prevalence of MetS increased $27 \%$ in men and $21 \%$ in women with the highest TSH quartile. Inconsistently, Mehran et al ${ }^{16}$ found no correlation between TSH within normal range and MetS. The disappearance of the correlation may be related to the exclusion of diabetic patients from the analysis and different criteria of MetS they selected. Huang et al reported no association between TSH and MetS after stratified analyses according to sex. ${ }^{17}$ The inconsistent results may be attributed to races, population, methodology, definitions of MetS and the varying set points of TSH for "euthyroid status". The two major criteria by the National Cholesterol Education Program Adult Treatment Panel III (NCEP ATP III) and the International Diabetes Federation (IDF) were widely used.
A

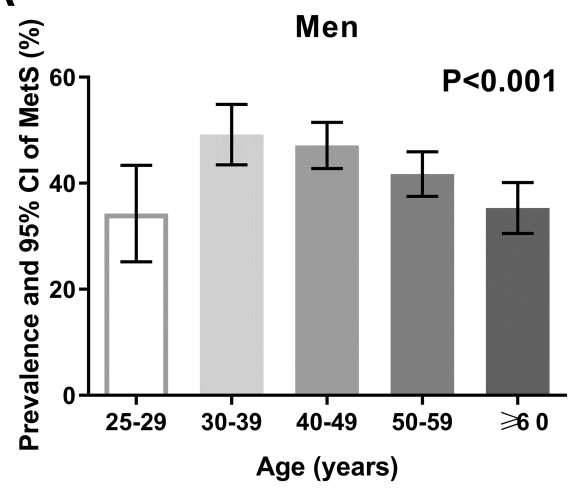

B

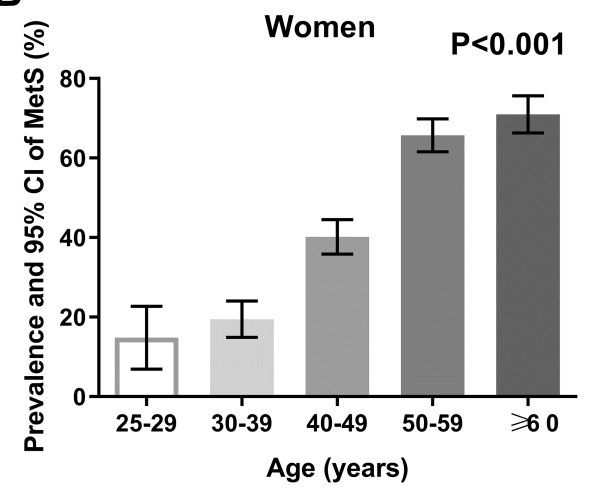

Figure I Prevalence of metabolic syndrome (MetS) and $95 \%$ confidence interval $(\mathrm{Cl})$ according to age in men $(\mathbf{A})$ and women (B).

Notes: $P$-value for the difference among age groups using a chi-squared test. 

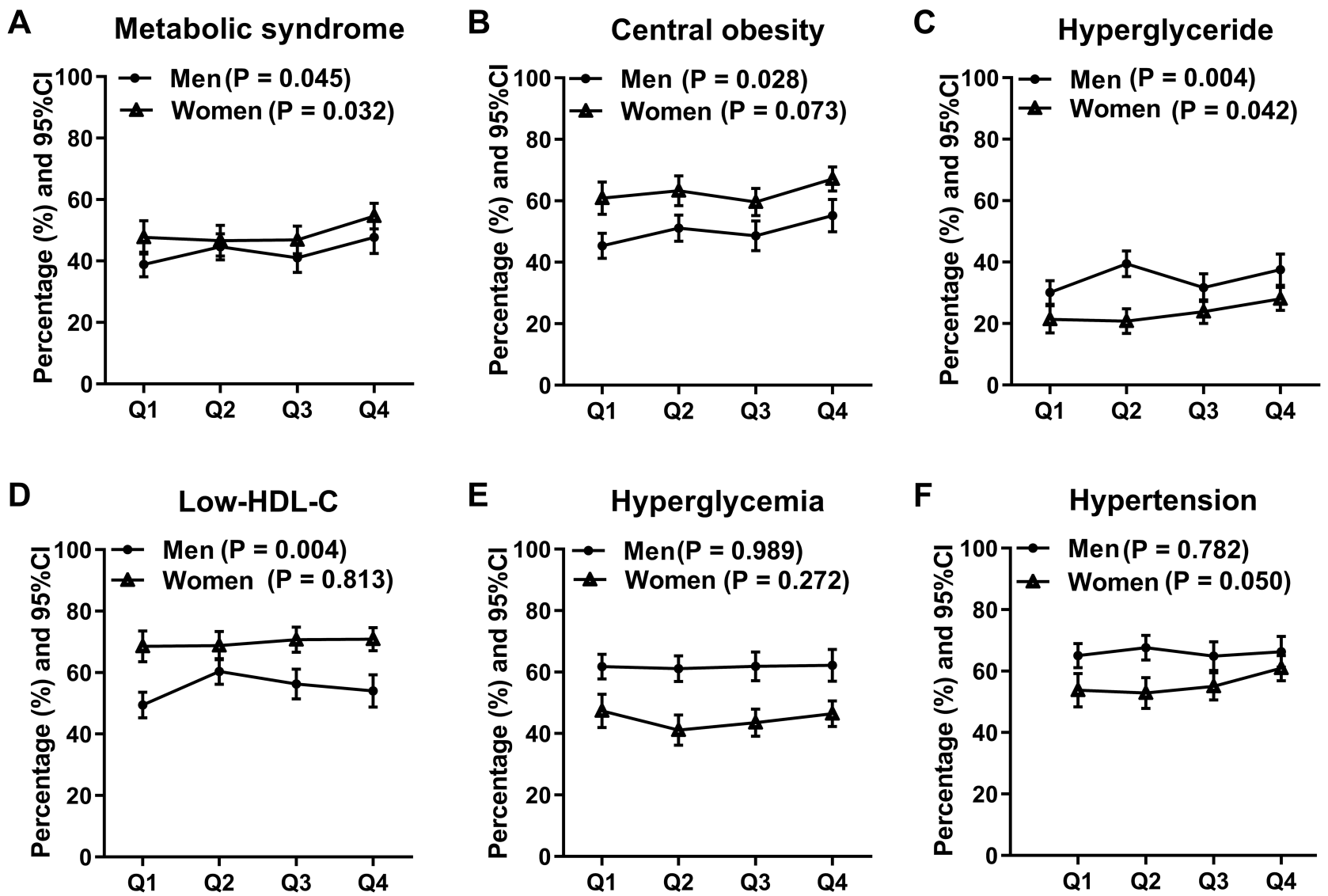

Figure 2 Percentage of metabolic disorders in men and women according to thyrotropin (TSH) quartiles.

Notes: QI-4 indicate the TSH quartiles. P-value for the difference among the TSH quartiles using a chi-squared test. (A) Metabolic syndrome. (B) Central obesity. (C) Hyperglyceride. (D) Low-HDL-C. (E) Hyperglycemia. (F) Hypertension.

Abbreviation: HDL-C, high-density lipoprotein cholesterol.

Here, we prefer to choose the IDF 2005 MetS criteria because it provides a specific diagnostic cut-point for Chinese population. ${ }^{24}$ This standard has also been adopted and confirmed by many studies from China. ${ }^{14,28}$ Notably, most of the previous studies did not give sufficient consideration to the potential confounding factors, including sex, age, lifestyle and serum levels of thyroid hormone, such as FT3 and FT4, which may be related to metabolic disease. ${ }^{29,30}$ Moreover, given that the occurrence of metabolic disease is characterized by gender dimorphism, we conducted analyses for men and women separately or adjusted for sex in all of the analysis models. Here we found that serum TSH was independently associated with the prevalence of MetS both in men and women after adjusting for the influence factors (including age, the intake frequency of red meat, smoking, drinking, sedentary time, and serum levels of FT4, and FT3).

Amongst the parameters of the metabolic syndrome, we also found TSH was correlated significantly with obesity and dyslipidemia. In euthyroidism, many cross-sectional studies showed the association between TSH and obesity. ${ }^{10,15,26,31,32}$ Also besides, some longitudinal studies have established the relationship between the concentrations of thyroid hormones and changes in body weight over time. ${ }^{3-35}$ In contrast, several studies did not detect the relation between TSH and obesity. $^{36,37}$ The variability of the maximum value in the reference range of TSH, limited sample size and the adjusting confounders might explain the differences of association between BMI and TSH. Besides adiposity, thyroid function also affected lipid metabolism. ${ }^{11,13,38,39}$ The HUNT study found a linear and significant increase in serum TC, LDL$\mathrm{C}$, TG levels, and a linear decrease in HDL-C with increasing TSH in euthyroid subjects. ${ }^{11}$ Similar results were observed in the PG population. There are controversial findings of the relationship between TSH and blood pressure in euthyroid subjects. $^{12,40,41}$ Here we found that TSH was just significantly correlated with blood pressure in women. Higher TSH levels within the normal range were not significantly associated with prediabetes or diabetes in most studies, ${ }^{16,27}$ 
Table 2 Association of TSH with Metabolic Syndrome by the Poisson Regression

\begin{tabular}{|c|c|c|c|c|c|c|c|}
\hline \multirow[t]{2}{*}{ Items } & \multirow{2}{*}{$\begin{array}{l}\text { TSH } \\
\text { Quartiles }\end{array}$} & \multicolumn{3}{|l|}{ Men } & \multicolumn{3}{|l|}{ Women } \\
\hline & & $\begin{array}{l}\text { Model I PR } \\
(95 \% \mathrm{CI})\end{array}$ & $\begin{array}{l}\text { Model } 2 \text { PR } \\
(95 \% \mathrm{Cl})\end{array}$ & $\begin{array}{l}\text { Model } 3 \text { PR } \\
(95 \% \mathrm{Cl})\end{array}$ & $\begin{array}{l}\text { Model I PR } \\
(95 \% \mathrm{CI})\end{array}$ & $\begin{array}{l}\text { Model } 2 \text { PR } \\
(95 \% \mathrm{Cl})\end{array}$ & $\begin{array}{l}\text { Model } 3 \text { PR } \\
(95 \% \mathrm{Cl})\end{array}$ \\
\hline \multirow[t]{3}{*}{ MetS } & Q2 & $1.15(1.00,1.32)$ & $1.14(0.99,1.31)$ & $1.14(1.00,1.32)$ & $0.98(0.84,1.14)$ & $1.03(0.90,1.19)$ & $1.05(0.91,1.20)$ \\
\hline & Q3 & $1.06(0.91,1.24)$ & $1.06(0.91,1.24)$ & $1.07(0.92,1.25)$ & $0.98(0.85,1.14)$ & $1.08(0.94,1.24)$ & $1.11(0.97,1.27)$ \\
\hline & Q4 & $1.25(1.08,1.46)^{* *}$ & $1.23(1.06,1.43)^{* *}$ & $1.27(1.09,1.48)^{* *}$ & $1.15(1.00,1.31)$ & $1.17(1.04,1.33)^{*}$ & $1.21(1.07,1.37)^{* * *}$ \\
\hline \multirow[t]{3}{*}{ Central obesity } & Q2 & $1.13(1.00,1.28)$ & $1.12(0.99,1.26)$ & $1.12(1.00,1.27)$ & $1.04(0.93,1.17)$ & $1.09(0.98,1.2 \mathrm{I})$ & $1.10(0.98,1.22)$ \\
\hline & Q3 & $1.07(0.94,1.23)$ & $1.08(0.95,1.23)$ & $1.09(0.95,1.24)$ & $0.98(0.87,1.10)$ & $1.03(0.93,1.15)$ & $1.05(0.94,1.17)$ \\
\hline & Q4 & $1.22(1.07,1.39)^{* *}$ & $1.23(1.08,1.40)^{* *}$ & $1.25(1.10,1.43)^{* * *}$ & $1.10(0.99,1.22)$ & $1.12(1.02,1.24)^{*}$ & $1.14(1.03,1.26)^{* *}$ \\
\hline \multirow[t]{3}{*}{ Hypertriglyceride } & Q2 & $1.31(1.11,1.55)^{* *}$ & $1.30(1.10,1.52)^{* *}$ & $1.30(1.11,1.15)^{* *}$ & $0.97(0.73,1.29)$ & $1.04(0.78,1.37)$ & $1.06(0.8 I, I .4 I)$ \\
\hline & Q3 & $1.05(0.87,1.27)$ & $1.09(0.90,1.31)$ & $1.10(0.91,1.32)$ & I.II $(0.86,1.45)$ & $1.23(0.95,1.59)$ & $1.30(1.00,1.68)^{*}$ \\
\hline & Q4 & $1.25(1.03,1.50)^{*}$ & $1.33(1.11,1.59)^{* *}$ & $1.38(1.15,1.66)^{* *}$ & $1.31(1.02,1.68)^{*}$ & $1.33(1.05,1.69)^{*}$ & $1.43(1.12,1.82)^{* *}$ \\
\hline \multirow[t]{3}{*}{ Low-HDL-C } & Q2 & $1.22(1.09,1.36)^{* *}$ & $1.23(1.10,1.36)^{* *}$ & $1.23(1.11,1.37)^{* *}$ & $1.00(0.91,1.11)$ & $1.01(0.92,1.12)$ & $1.02(0.92,1.12)$ \\
\hline & Q3 & $1.14(1.01,1.28)^{*}$ & $1.15(1.02,1.29)^{*}$ & $1.15(1.02,1.30)^{*}$ & $1.03(0.94,1.13)$ & $1.05(0.96,1.15)$ & $1.05(0.96,1.16)$ \\
\hline & Q4 & $1.09(0.96,1.24)$ & $1.12(0.99,1.27)$ & $1.14(1.00,1.29)$ & $1.04(0.95,1.13)$ & $1.04(0.95,1.14)$ & $1.04(0.95,1.14)$ \\
\hline \multirow[t]{3}{*}{ Hypertension } & Q2 & $1.04(0.96,1.13)$ & $1.03(0.95,1.12)$ & $1.04(0.96,1.13)$ & $0.98(0.86,1.13)$ & $1.03(0.92,1.17)$ & $1.05(0.93,1.19)$ \\
\hline & Q3 & $1.00(0.91,1.10)$ & $0.98(0.89,1.06)$ & $0.99(0.90,1.08)$ & $1.02(0.90,1.16)$ & $1.13(1.01,1.27)^{*}$ & $1.16(1.04,1.30)^{*}$ \\
\hline & Q4 & $1.02(0.93,1.12)$ & $0.96(0.88,1.06)$ & $0.99(0.90,1.09)$ & $1.13(1.00,1.28)^{*}$ & $1.16(1.04,1.29)^{* *}$ & $1.20(1.08,1.33)^{* *}$ \\
\hline \multirow[t]{3}{*}{ Hyperglycemia } & Q2 & $1.01(0.91,1.12)$ & $0.99(0.90,1.08)$ & $0.99(0.90,1.09)$ & $0.87(0.74,1.02)$ & $0.92(0.79,1.06)$ & $0.94(0.81,1.09)$ \\
\hline & Q3 & $1.00(0.91,1.11)$ & $0.99(0.89,1.09)$ & $0.99(0.90,1.10)$ & $0.92(0.79,1.07)$ & $1.03(0.89,1.18)$ & $1.07(0.93,1.23)$ \\
\hline & Q4 & $0.99(0.90,1.09)$ & $0.96(0.86,1.06)$ & $0.97(0.88,1.08)$ & $0.98(0.85,1.13)$ & $1.00(0.87,1.14)$ & $1.05(0.92,1.20)$ \\
\hline
\end{tabular}

Notes: QI-4 were grouped according to TSH quartiles. Model I: unadjusted. Model 2: adjusted for age, smoking, drinking, red meat intake, sedentary time and menopause (in women). Model 3: further adjusted for free triiodothyronine (FT3), and free thyroxine (FT4). $P$-value $<0.05$ was considered significant. Significant level: $* P$-value $<0.05$; **P-value $<0.01$ by Poisson regression analysis.

Abbreviations: PR, prevalence ratio; TSH, thyrotropin; Q, quartile; MetS, metabolic syndrome; HDL-C, high-density lipoprotein cholesterol.

which was consistent with our observations. One of the explanations for the lack of correlation between TSH in the normal range and blood glucose is the effect of thyroid hormone on promoting hepatic glucogenesis. ${ }^{42}$

The mechanism of the relationship between thyroid function and MetS does not appear to be well established. Thyroid hormones have essential physiologic roles in energy homeostasis and metabolism, and cardiovascular function. Individuals with high TSH levels within the normal range, had a higher risk of MetS and the components including obesity, dyslipidemia, and hypertension. A proinflammatory state probably contributes to the syndrome. ${ }^{43-45}$ Obesity is an important element of MetS, and TSH is demonstrated to be independently associated with obesity in our and others' studies. TSH receptors are widely expressed in adipose tissue and this signaling pathway has been implicated in adipogenesis processes, suggesting that TSH itself may stimulate adipogenesis. ${ }^{46-48}$ Leptin, secreted by adipocyte, has also been shown to stimulate the transcription of pro-thyrotropin-releasing hormone (TRH) and consequently also that of $\mathrm{TSH}^{49-51}$ A recent study has shown that leptin and adiponectin had an addictive and independent effect on the association between thyroid hormones and the components of MetS. ${ }^{52}$ It has also been demonstrated that TSH promoted cholesterol synthesis in the liver and stimulated lipolysis in cultured adipocytes and the elevation of serum-free fatty acid levels in vivo. ${ }^{53,54}$ Some studies found that TSH was also related to insulin resistance, ${ }^{55}$ which was an important mediator for the effect of thyroid function on MetS. ${ }^{14}$ In our study, we found that TSH was correlated with HOMA-IR, but this relation no longer remained significant after adjustment for age.

The strength of the present study is that we performed the analysis based on a large-scale community-based Chinese population, and evaluated the association between TSH and MetS stratified by gender before and after adjustment for the potential confounders. However, despite well-characterized covariate data allowing us to perform multivariable-adjusted analyses, the generalizability of the results needs to be cautious. In addition, as a cross-sectional study, we cannot infer causality on the association between TSH and MetS in euthyroid population. More longitudinal cohort studies and randomized clinical trials are to be warranted to confirm these associations. 


\section{Conclusion}

In the present study, we found that the prevalence of MetS and its most components increased in participants with higher TSH quartile group in euthyroid population. The assessment of thyroid function may contribute to early identification and treatment of chronic metabolic diseases.

\section{Ethical Approval and Informed Consent}

The study was approved by the ethics committee of Peking University Health Science Center (No. IRB0000105212022). All procedures in the study were performed in accordance with the ethical standards of the Declaration of Helsinki. All participants gave written informed consent.

\section{Acknowledgments}

We thank all the participants for agreeing to join the PMDS. We also thank all the investigators of Beijing Pinggu Hospital for their contribution. The authors would like to thank Mrs Xian Li from the George Institute for Global Health, China, for her kind support with the statistical models.

\section{Disclosure}

The authors report no conflicts of interest in this work.

\section{References}

1. Samson SL, Garber AJ. Metabolic syndrome. Endocrinol Metab Clin North Am. 2014;43(1):1-23. doi:10.1016/j.ecl.2013.09.009

2. Eckel RH, Grundy SM, Zimmet PZ. The metabolic syndrome. Lancet. 2005;365(9468):1415-1428. doi:10.1016/S0140-6736(05) 66378-7

3. Kim B. Thyroid hormone as a determinant of energy expenditure and the basal metabolic rate. Thyroid. 2008;18(2):141-144. doi:10.1089/ thy.2007.0266

4. Razvi S, Jabbar A, Pingitore A, et al. Thyroid hormones and cardiovascular function and diseases. J Am Coll Cardiol. 2018;71(16):17811796. doi:10.1016/j.jacc.2018.02.045

5. Wang B, Song R, He W, et al. Sex differences in the associations of obesity with hypothyroidism and thyroid autoimmunity among chinese adults. Front Physiol. 2018;9:1397. doi:10.3389/fphys.2018.01397

6. Maratou E, Hadjidakis DJ, Kollias A, et al. Studies of insulin resistance in patients with clinical and subclinical hypothyroidism. Eur $J$ Endocrinol. 2009;160(5):785-790. doi:10.1530/EJE-08-0797

7. Sgarbi JA, Matsumura LK, Kasamatsu TS, Ferreira SR, Maciel RMB. Subclinical thyroid dysfunctions are independent risk factors for mortality in a 7.5-year follow-up: the Japanese-Brazilian thyroid study. Eur $J$ Endocrinol. 2010;162(3):569-577. doi:10.1530/EJE-09-0845

8. Asvold BO, Bjøro T, Platou C, Vatten LJ. Thyroid function and the risk of coronary heart disease: 12-year follow-up of the HUNT study in Norway. Clin Endocrinol (Oxf). 2012;77(6):911-917. doi:10.1111/ j.1365-2265.2012.04477.x
9. Rotondi M, Magri F, Chiovato L. Risk of coronary heart disease and mortality for adults with subclinical hypothyroidism. JAMA. 2010;304(22):2481-2482. doi:10.1001/jama.2010.1786

10. Knudsen N, Laurberg P, Rasmussen LB, et al. Small differences in thyroid function may be important for body mass index and the occurrence of obesity in the population. J Clin Endocrinol Metab. 2005;90(7):4019-4024. doi:10.1210/jc.2004-2225

11. Asvold BO, Vatten LJ, Nilsen TIL, Bjøro T. The association between TSH within the reference range and serum lipid concentrations in a population-based study. The HUNT study. Eur $J$ Endocrinol. 2007;156(2):181-186. doi:10.1530/eje.1.02333

12. Asvold BO, Bjøro T, Nilsen TIL, Vatten LJ. Association between blood pressure and serum thyroid-stimulating hormone concentration within the reference range: a population-based study. $J$ Clin Endocrinol Metab. 2007;92(3):841-845. doi:10.1210/jc.2006-2208

13. Shinkov A, Borissova A-M, Kovatcheva R, Atanassova I, Vlahov J, Dakovska L. The prevalence of the metabolic syndrome increases through the quartiles of thyroid stimulating hormone in a populationbased sample of euthyroid subjects. Arq Bras Endocrinol Metabol. 2014;58(9):926-932. doi:10.1590/0004-2730000003538

14. Chang Y-C, Hua S-C, Chang C-H, et al. High TSH level within normal range is associated with obesity, dyslipidemia, hypertension, inflammation, hypercoagulability, and the metabolic syndrome: a novel cardiometabolic marker. J Clin Med. 2019;8(6):817. doi:10. $3390 / \mathrm{jcm} 8060817$

15. Ruhla S, Weickert MO, Arafat AM, et al. A high normal TSH is associated with the metabolic syndrome. Clin Endocrinol (Oxf). 2010;72(5):696-701. doi:10.1111/j.1365-2265.2009.03698.x

16. Mehran L, Amouzegar A, Tohidi M, Moayedi M, Azizi F. Serum free thyroxine concentration is associated with metabolic syndrome in euthyroid subjects. Thyroid. 2014;24(11):1566-1574. doi:10.1089/ thy.2014.0103

17. Huang CY, Hwang LC. The association of thyroid hormones and Tsh with the metabolic syndrome in euthyroid Taiwanese individuals. Endocr Pract. 2016;22(11):1303-1309. doi:10.4158/EP161260.OR

18. Zhang J, Jiang R, Li L, et al. Serum thyrotropin is positively correlated with the metabolic syndrome components of obesity and dyslipidemia in chinese adolescents. Int J Endocrinol. 2014;2014:289503. doi: $10.1155 / 2014 / 289503$

19. Ren R, Ma Y, Deng F, et al. Association between serum TSH levels and metabolic components in euthyroid subjects: a nationwide population-based study. Diabetes Metab Syndr Obes. 2019;12:1563-1569. doi:10.2147/DMSO.S202769

20. Lai Y, Wang J, Jiang F, et al. The relationship between serum thyrotropin and components of metabolic syndrome. Endocr $J$. 2011;58(1):23-30. doi:10.1507/endocrj.K10E-272

21. Zhou X, Li Y, Zhang X, et al. Independent markers of nonalcoholic fatty liver disease in a gentrifying population-based Chinese cohort. Diabetes Metab Res Rev. 2019;35(5):e3156-e3156. doi:10.1002/dmrr.3156

22. Zhang X, Li Y, Zhou X, Han X, Gao Y, Ji L. Association between serum thyrotropin within the euthyroid range and obesity. Endocr $J$. 2019;66(5):451-457. doi:10.1507/endocrj.EJ18-0140

23. Du H, Bennett D, Li L, et al. Physical activity and sedentary leisure time and their associations with BMI, waist circumference, and percentage body fat in 0.5 million adults: the China Kadoorie Biobank study. Am J Clin Nutr. 2013;97(3):487-496. doi:10.3945/ ajcn.112.046854

24. Alberti KGMM, Zimmet P, Shaw J. Metabolic syndrome-a new world-wide definition. A consensus statement from the international diabetes federation. Diabet Med. 2006;23(5):469-480. doi:10.1111/ j.1464-5491.2006.01858.x

25. Zhou B-F. Cooperative meta-analysis group of the working group on obesity in C. Predictive values of body mass index and waist circumference for risk factors of certain related diseases in Chinese adultsstudy on optimal cut-off points of body mass index and waist circumference in Chinese adults. Biomed Environ Sci. 2002;15(1):83-96. 
26. Oh JY, Sung YA, Lee HJ. Elevated thyroid stimulating hormone levels are associated with metabolic syndrome in euthyroid young women. Korean J Intern Med. 2013;28(2):180-186. doi:10.3904/ kjim.2013.28.2.180

27. Zhou Y-C, Fang W-H, Kao T-W, et al. Exploring the association between thyroid- stimulating hormone and metabolic syndrome: a large population-based study. PLoS One. 2018;13(6):e0199209. doi:10.1371/journal.pone.0199209

28. Liu C, Scherbaum WA, Schott M, Schinner S. Subclinical hypothyroidism and the prevalence of the metabolic syndrome. Horm Metab Res. 2011;43(6):417-421. doi:10.1055/s-0031-1275719

29. de Oliveira Otto MC, Alonso A, Lee D-H, et al. Dietary intakes of zinc and heme iron from red meat, but not from other sources, are associated with greater risk of metabolic syndrome and cardiovascular disease. J Nutr. 2012;142(3):526-533. doi:10.3945/jn.111.149781

30. Urrunaga-Pastor D, Guarnizo-Poma M, Moncada-Mapelli E, et al. High free triiodothyronine and free-triiodothyronine-to-free-thyroxine ratio levels are associated with metabolic syndrome in a euthyroid population. Diabetes Metab Syndr. 2018;12(2):155-161. doi:10.1016/ j.dsx.2017.12.003

31. Sakurai M, Nakamura K, Miura K, et al. Association between a serum thyroid-stimulating hormone concentration within the normal range and indices of obesity in Japanese men and women. Intern Med. 2014;53(7):669-674. doi:10.2169/internalmedicine.53.1387

32. Asvold BO, Bjøro T, Vatten LJ. Association of serum TSH with high body mass differs between smokers and never-smokers. J Clin Endocrinol Metab. 2009;94(12):5023-5027. doi:10.1210/jc.20091180

33. Svare A, Nilsen TIL, Bjøro T, Asvold BO, Langhammer A. Serum TSH related to measures of body mass: longitudinal data from the HUNT study, Norway. Clin Endocrinol (Oxf). 2011;74(6):769-775. doi:10.1111/j.1365-2265.2011.04009.x

34. Nyrnes A, Jorde R, Sundsfjord J. Serum TSH is positively associated with BMI. Int J Obes (Lond). 2006;30(1):100-105. doi:10.1038/sj. ijo.0803112

35. Bjergved L, Jørgensen T, Perrild H, et al. Thyroid function and body weight: a community-based longitudinal study. PLoS One. 2014;9(4): e93515-e93515. doi:10.1371/journal.pone.0093515

36. Makepeace AE, Bremner AP, O'Leary P, et al. Significant inverse relationship between serum free $\mathrm{T} 4$ concentration and body mass index in euthyroid subjects: differences between smokers and nonsmokers. Clin Endocrinol (Oxf). 2008;69(4):648-652. doi:10.1111/ j.1365-2265.2008.03239.x

37. Manji N, Boelaert K, Sheppard MC, Holder RL, Gough SC, Franklyn JA. Lack of association between serum TSH or free T4 and body mass index in euthyroid subjects. Clin Endocrinol (Oxf). 2006;64 (2):125-128. doi:10.1111/j.1365-2265.2006.02433.x

38. Wang F, Tan Y, Wang C, et al. Thyroid-stimulating hormone levels within the reference range are associated with serum lipid profiles independent of thyroid hormones. J Clin Endocrinol Metab. 2012;97 (8):2724-2731. doi:10.1210/jc.2012-1133

39. Roos A, Bakker SJL, Links TP, Gans ROB, Wolffenbuttel BHR. Thyroid function is associated with components of the metabolic syndrome in euthyroid subjects. J Clin Endocrinol Metab. 2007;92 (2):491-496. doi:10.1210/jc.2006-1718
40. Gu Y, Zheng L, Zhang Q, et al. Relationship between thyroid function and elevated blood pressure in euthyroid adults. $J$ Clin Hypertens. 2018;20(10):1541-1549. doi:10.1111/jch.13369

41. Liu D, Jiang F, Shan Z, et al. A cross-sectional survey of relationship between serum TSH level and blood pressure. J Hum Hypertens. 2010;24(2):134-138. doi:10.1038/jhh.2009.44

42. Sinha RA, Singh BK, Yen PM. Thyroid hormone regulation of hepatic lipid and carbohydrate metabolism. Trends Endocrinol Metab. 2014;25(10):538-545. doi:10.1016/j.tem.2014.07.001

43. Sutherland JP, McKinley B, Eckel RH. The metabolic syndrome and inflammation. Metab Syndr Relat Disord. 2004;2(2):82-104. doi:10.1089/met.2004.2.82

44. Trayhurn P, Wood IS. Adipokines: inflammation and the pleiotropic role of white adipose tissue. Br J Nutr. 2004;92(3):347-355. doi:10. 1079/BJN20041213

45. Weisberg SP, McCann D, Desai M, Rosenbaum M, Leibel RL, Ferrante AW Jr. Obesity is associated with macrophage accumulation in adipose tissue. J Clin Invest. 2003;112(12):1796-1808. doi:10.11 72/JCI200319246

46. Santini F, Marzullo P, Rotondi M, et al. Mechanisms in endocrinology: the crosstalk between thyroid gland and adipose tissue: signal integration in health and disease. Eur J Endocrinol. 2014;171(4): R137-R152. doi:10.1530/EJE-14-0067

47. Reinehr T. Obesity and thyroid function. Mol Cell Endocrinol. 2010;316(2):165-171. doi:10.1016/j.mce.2009.06.005

48. Winter WE, Signorino MR. Review: molecular thyroidology. Ann Clin Lab Sci. 2001;31(3):221-244.

49. Menendez C, Baldelli R, Camiña JP, et al. TSH stimulates leptin secretion by a direct effect on adipocytes. J Endocrinol. 2003;176 (1):7-12. doi:10.1677/joe.0.1760007

50. Mantzoros CS, Magkos F, Brinkoetter M, et al. Leptin in human physiology and pathophysiology. Am J Physiol Endocrinol Metab. 2011;301(4):E567-E584. doi:10.1152/ajpendo.00315.2011

51. Dietlein M, Kahaly G, Kobe C, Schmidt M, Derwahl KM, Schicha H. Obesity, energy regulation and thyroid function: is borderline elevated TSH-level the cause or secondary phenomenon of obesity. Nuklearmedizin. 2008;47(5):181-187. doi:10.3413/nukmed-0175

52. Delitala AP, Scuteri A, Fiorillo E, Lakatta EG, Schlessinger D, Cucca F. Role of adipokines in the association between thyroid hormone and components of the metabolic syndrome. J Clin Med. 2019;8:6. doi: $10.3390 / \mathrm{jcm} 8060764$

53. Tian L, Song Y, Xing M, et al. A novel role for thyroid-stimulating hormone: up-regulation of hepatic 3-hydroxy-3-methyl-glutarylcoenzyme A reductase expression through the cyclic adenosine monophosphate/protein kinase $\mathrm{A} /$ cyclic adenosine monophosphateresponsive element binding protein pathway. Hepatology. 2010;52 (4):1401-1409. doi:10.1002/hep. 23800

54. Gagnon A, Antunes TT, Ly T, et al. Thyroid-stimulating hormone stimulates lipolysis in adipocytes in culture and raises serum free fatty acid levels in vivo. Metabolism. 2010;59(4):547-553. doi:10.1016/j.metabol.2009.08.018

55. Benseñor IM, Goulart AC, Molina M, Peixoto de Miranda ÉJ, Santos IS, Lotufo PA. Thyrotropin levels, insulin resistance, and metabolic syndrome: a cross-sectional analysis in the Brazilian longitudinal study of adult health (ELSA-Brasil). Metab Syndr Relat Disord. 2015;13(8):362-369. doi:10.1089/met.2015.0045 


\section{Publish your work in this journal}

Diabetes, Metabolic Syndrome and Obesity: Targets and Therapy is an international, peer-reviewed open-access journal committed to the rapid publication of the latest laboratory and clinical findings in the fields of diabetes, metabolic syndrome and obesity research. Original research, review, case reports, hypothesis formation, expert opinion and commentaries are all considered for publication. The manuscript management system is completely online and includes a very quick and fair peer-review system, which is all easy to use. Visit http://www.dovepress.com/testimonials.php to read real quotes from published authors. 